\title{
Resistance maps from local probing of a ballistic mesoscopic Hall bar
}

\author{
G. Papp ${ }^{\text {a) }}$ \\ Department of Theoretical Physics, University of Szeged, Tisza L. krt. 84-86, H-6720 Szeged, Hungary and \\ Institute of Physics, University of West Hungary, Bajcsy Zs. út 5-7, H- 9400 Sopron, Hungary \\ F. M. Peeters \\ Departement Fysica, Universiteit Antwerpen (CGB), Groenenborgerlaan 171, B-2020 Antwerpen, Belgium
}

(Received 26 August 2006; accepted 22 January 2007; published online 30 March 2007)

\begin{abstract}
The response of a ballistic mesoscopic Hall bar to a local potential barrier is investigated in the presence of an external magnetic field. The effect of the position of the local potential probe in the Hall cross on the Hall and bend resistance is investigated leading to two-dimensional (2D) resistance maps. The Onsager-Casimir symmetry relations and symmetry relations between resistance measurements when interchanging the current and voltage leads, is obtained numerically for such 2D resistance maps. Symmetry properties are derived for the Hall and bend resistance maps with respect to the position of the local potential barrier. (C) 2007 American Institute of Physics.
\end{abstract} [DOI: 10.1063/1.2713365]

\section{INTRODUCTION}

In present day microelectronics the determination of local electric fields is of paramount importance. Such fields influence the conductance properties of the device and its operating characteristics. Using the nanopotentiometry technique it has been demonstrated that the distribution of the electrical potential on a surface ${ }^{1,2}$ can be mapped. One uses a conductive atomic force microscope (AFM) tip in contact, ${ }^{1}$ or noncontact ${ }^{2}$ mode, as a local voltage probe to map the local potential landscape. The latter determines the electron scattering and consequently the electrical resistance. On the other hand, such tips can also be used to induce potential variations in the sample in order to influence the conductance locally. ${ }^{3}$ Measuring the change in the resistance of the device gives information on the local transport properties and tells us how effective such a local perturbation is in disturbing the electron motion. In small devices as, e.g., a mesoscopic Hall bar the current is non homogeneously distributed over the sample and as a consequence the effect of a local potential will depend on its spatial position. The Hall resistance and longitudinal resistance of a Hall bar were recently measured ${ }^{4}$ while an AFM tip scanned at constant height over the Hall cross. The measurements showed a characteristic distribution of the Hall and longitudinal resistances which is a measure of the sensitivity of the Hall bar to a local potential.

Ballistic transport of electrons in a cross-shaped Hall junction placed in a homogeneous magnetic field, containing a local potential profile in the cross junction was studied by Baelus and Peeters ${ }^{5}$ using a semiclassical formalism for electron transport. They demonstrated theoretically ${ }^{5}$ that the twodimensional electron gas could be used as a probe to measure the induced potential profile. This potential profile was fixed at the center of the Hall cross. Here we relax this constraint and obtain a full two-dimensional (2D) map of the Hall bar resistances. Furthermore, we present a numerical check of the Onsager-Casimir ${ }^{6,7}$ symmetry relations and

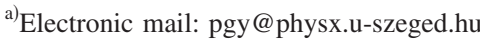

confirm the symmetry relations between conductance measurements when interchanging the current and voltage leads of the Hall bar (see Fig. 1) as predicted theoretically by Büttiker ${ }^{8}$ for a four terminal conductor but now for the case that a local potential profile, as created by a scanning AFM tip, is present. We also find symmetry relations for the resistance maps.

\section{MODEL}

In order to calculate the Hall and the bend resistances of the Hall cross, we will use the billiard model ${ }^{9}$ which was proven to be applicable in the ballistic regime. The electrons are considered as point particles which is justified when the Fermi wavelength $\lambda_{F} \ll W, d$ where $2 W$ is the width of the Hall probes and $2 d$ is the half width of the potential profile which acts as a scatterer for the electrons. We assume that temperature is not extremely low such that interference effects are averaged out due to thermal smearing. This temperature smearing will make the condition $\lambda_{F} \ll W, d$ less restrictive. The electron motion is governed by Newton's law, which is justified at low temperatures and in the case of high mobility samples where the mean-free path $\ell_{e} \gg W, d$. In a typical $\mathrm{GaAs}$ heterostructure the electron density is $n$ $\sim 3 \times 10^{11} \mathrm{~cm}^{-2}$ with a typical low temperature mobility $\mu$ $\sim 10^{6} \mathrm{~cm}^{2} / \mathrm{V} \mathrm{s}$, which gives $\lambda_{F}=450 \AA$ and $\ell_{e}=5.4 \mu \mathrm{m}$. This billiard model was applied ${ }^{9}$ and described, e.g., the experiments of Ref. 10 and explained ${ }^{11}$ the response of the ballistic magnetometer. ${ }^{12}$

Applying the Landauer-Büttiker formalism ${ }^{8}$ for the four terminal conductor with identical leads, the $R_{\alpha}=\left(R_{31,24}\right.$ $\left.+R_{24,31}\right) / 2, \quad R_{\beta}=\left(R_{14,32}+R_{32,14}\right) / 2, \quad R_{\gamma}=\left(R_{12,43}+R_{43,12}\right) / 2$ resistances fulfill the $R_{\alpha}+R_{\beta}+R_{\gamma}=0$ relation and contains the $R_{H}$ Hall resistance

$$
R_{H}=R_{31,24}=\left(h / e^{2}\right)\left(T_{23} T_{41}-T_{21} T_{43}\right) / D,
$$

and the $R_{B}$ bend resistance 


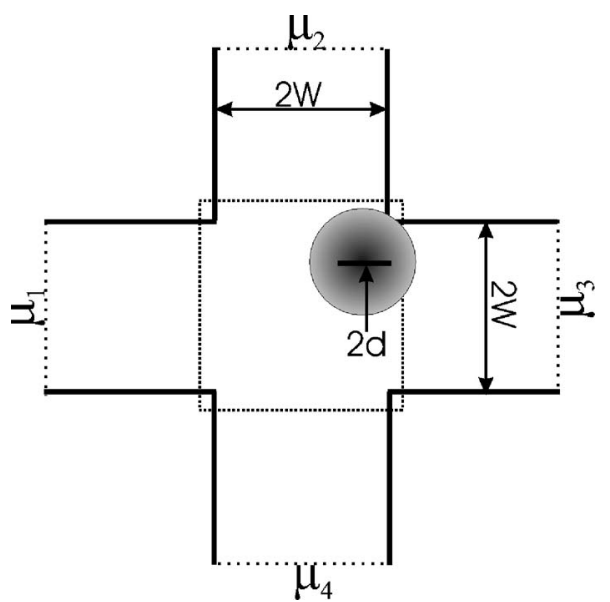

FIG. 1. The Hall cross with the four reservoirs at chemical potentials $\mu_{1}, \mu_{2}$, $\mu_{3}$, and $\mu_{4}$. A potential barrier is applied (shaded area) and the dotted square indicates the scanning area considered in the calculations.

$$
R_{B}=R_{14,32}=\left(h / e^{2}\right)\left(T_{31} T_{24}-T_{34} T_{21}\right) / D,
$$

where $D=\left(h / e^{2}\right)^{2}\left(\alpha_{11} \alpha_{22}-\alpha_{12} \alpha_{21}\right) S$ with $S=T_{12}+T_{14}+T_{32}$ $+T_{34}=T_{21}+T_{41}+T_{23}+T_{43}, \quad \alpha_{11}=\left(e^{2} / h\right)\left[\left(1-T_{11}\right) S-\left(T_{14}+T_{12}\right)\right.$ $\left.\times\left(T_{41}+T_{21}\right)\right] / S, \alpha_{12}=\left(e^{2} / h\right)\left(T_{12} T_{34}-T_{14} T_{32}\right) / S, \quad \alpha_{22}=\left(e^{2} / h\right)$ $\times\left[\left(1-T_{22}\right) S-\left(T_{21}+T_{23}\right)\left(T_{32}+T_{12}\right)\right] / S$, and $\alpha_{21}=\left(e^{2} / h\right)$ $\times\left(T_{21} T_{43}-T_{41} T_{23}\right) / S . T_{i j}(B)$ is the probability for carriers incident in the lead $j$ to be transmitted into the lead $i$. They satisfy the symmetry property: $T_{i j}(B)=T_{j i}(-B)$ which follows from current conservation and time-reversal invariance. This symmetry relation guarantees the reciprocity relation: $R_{m n, k l}(B)=R_{k l, m n}(-B)$, where $R_{m n, k l}=\left(\mu_{l}-\mu_{k}\right) / e I_{n m}$. So the reciprocity relation states that the resistance measured in the presence of a magnetic field $B$ is equal to the resistance measured in the presence of a reversed magnetic field $-B$ if the reversal is accompanied by an interchange of the current and voltage leads. The reciprocity relation follows from the Onsager-Casimir relation: ${ }^{6,7} \rho(B)=\rho^{T}(-B)$ for the resistivity tensor (here $T$ denotes the transpose).

The probabilities $T_{i j}(B)$ are calculated for the Hall bar using the ballistic billiard model. ${ }^{9,11}$ We inject a large number of electrons $\left(N_{e} \geq 10^{5}\right)$ toward the junction through, e.g., lead 1, and follow their classical trajectories to determine the probabilities: $T_{j 1}=N_{j} / N_{e}$, where $N_{j}$ is the number of electrons collected in lead $j$. Note that for the case of nonidentical leads or in the presence of an asymmetric local potential profile, similar procedures should be followed for each of the four leads. The electrons are injected uniformly over lead 1 , with Fermi velocity $v_{F}=\sqrt{2 m E_{F}}$, and angular distribution $P(\theta)=\cos (\theta) / 2$, where $\theta \in(-\pi / 2, \pi / 2)$ is the injection angle with respect to the channel axis. Here the angular distribution weight function $P(\Theta) \sim \cos (\Theta)$ simply results from the slight shift of the Fermi surface of the reservoirs in the linear response regime and the factor $1 / 2$ is due to normalization.

In the following we will express the magnetic field in units of $B_{0}=m v_{F} / 2 \mathrm{eW}$, and the resistance in $R_{0}$ $=\left(h / 2 e^{2}\right) \pi / 2 k_{F} W$, where $W$ is the half width of the leads, $m$ is the mass of the electron, $k_{F}=\sqrt{2 m E_{F} / \hbar^{2}}$, and $v_{F}=\hbar k_{F} / m$ the Fermi velocity. For electrons moving in GaAs ( $m$ $\left.=0.067 m_{e}\right)$ and for a typical channel width of $2 W=1 \mu \mathrm{m}$ and a Fermi energy of $E_{F}=13 \mathrm{meV}\left(n_{e}=3.65 \times 10^{11} \mathrm{~cm}^{-2}\right)$, we obtain $B_{0}=99 \mathrm{mT}$ and $R_{0}=270.5 \Omega$.

In order to demonstrate the main physics involved, we model the functional form of the probing potential by a Gaussian potential barrier: $V(\boldsymbol{\rho})=V_{0} \exp \left[-\left(\boldsymbol{\rho}-\boldsymbol{\rho}_{0}\right)^{2} / d^{2}\right]$ with height $V_{0}, 2 d$ width at half height and $\boldsymbol{\rho}_{0}$ is the center position of the potential on the Hall bar. This potential barrier is schematically shown in Fig. 1 by the shaded circular area. The effect of the potential barrier is to locally reduce the kinetic energy of the electrons to $E_{F}-V(\boldsymbol{\rho})$. Hence, the electron velocity $v$, the density $n$ of the electrons, and also the radius $R_{c}$ of the cyclotron orbit, are reduced by the potential. For $V_{0}<0$ a potential dip is induced in the Hall bar and the opposite occurs. The presence of the potential barrier will result in changes in the transmission probabilities $T_{i j}$ and the size of the change will result in, e.g., a Hall resistance $R_{H}\left(\boldsymbol{\rho}_{0}, B\right)$ which not only depends on the magnetic field but also on the position of the potential scatterer.

\section{RESULTS AND DISCUSSIONS}

First we demonstrate the Onsager relations for the case of a Hall bar when the tip is scanned over the Hall cross. Suppose the current flows from lead 1 to lead 3. The measured potentials are $\mu_{2}=e V_{2}$ and $\mu_{4}=e V_{4}$ under the condition that the current in leads 2 and 4 is zero. In this configuration the measured resistance is $R_{31,24}=\left(V_{4}-V_{2}\right) / I_{13}$. Now we switch the current and voltage leads but keep the magnetic field fixed. In this case the measured resistance is $R_{24,31}$ $=\left(V_{3}-V_{1}\right) / I_{24}$. We calculated those resistances as function of the tip position for: $V_{0} / E_{F}=0.5$ and $d / W=0.25$. Figure 2 shows contour plots of the resistances in the presence of an applied field of $B=50 \mathrm{mT}$ (left panels) and $B=-50 \mathrm{mT}$ (right panels). Since both $R_{31,24}$ (top panels) and $R_{24,31}$ (bottom panels) are off-diagonal Onsager coefficients their asymmetry is completely compatible with the Onsager symmetry relations. Furthermore, we find the following symmetry relations from Fig. 2:

$$
\begin{aligned}
R_{31,24}\left(x_{0}, y_{0} ; B\right) & =-R_{31,24}\left(-x_{0}, y_{0} ;-B\right) \\
& =-R_{31,24}\left(x_{0},-y_{0} ;-B\right), \\
R_{31,24}\left(x_{0}, y_{0} ; B\right) & =R_{31,24}\left(-x_{0},-y_{0} ; B\right) \\
& =R_{31,24}\left(-y_{0},-x_{0} ; B\right) \\
R_{31,24}\left(x_{0}, y_{0} ; B\right) & =R_{24,31}\left(x_{0}, y_{0} ;-B\right) .
\end{aligned}
$$

There are two additional possibilities of feeding two currents into the Hall cross of Fig. 1. Each of these four probes obeys the Onsager relations with a set of resistances which are the terms in $R_{\beta}$ and $R_{\gamma}$. Namely, the terms in $R_{\beta}$ are the bend resistances: $R_{14,32}=\left(\mu_{3}-\mu_{2}\right) / e I_{14}, \quad R_{32,14}=\left(\mu_{1}\right.$ $\left.-\mu_{4}\right) / e I_{32} ;$ and the terms in $R_{\gamma}$ are: $R_{12,43}=\left(\mu_{4}-\mu_{3}\right) / e I_{12}$, $R_{43,12}=\left(\mu_{1}-\mu_{2}\right) / e I_{43}$. These resistances are shown in Fig. 3 for the Hall bar with the same potential parameters as used in Fig. 2 for $B=50 \mathrm{mT}$. It is apparent that these figures exhibit the following symmetries: 

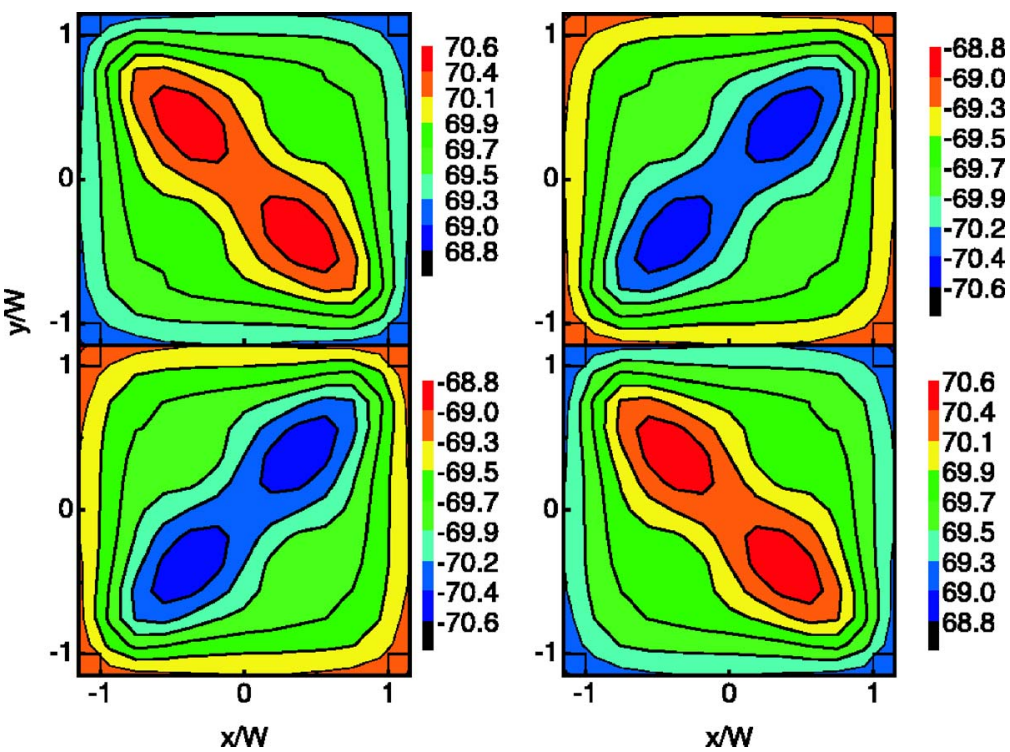

FIG. 2. (Color online) The Hall resistances: $R_{31,24}$ (top panels) and $R_{24,31}$ (bottom panels) for $B=50 \mathrm{mT}$ (left panels) and for $B=-50 \mathrm{mT}$ (right panels) with $V_{0} / E_{F}$ $=0.5$ and $d / W=0.25$. The value of the resistances is in $\Omega$.

$$
\begin{aligned}
R_{14,32}\left(x_{0}, y_{0} ; B\right) & =R_{32,14}\left(-x_{0},-y_{0} ; B\right), \\
& =-R_{12,43}\left(x_{0},-y_{0} ; B\right), \\
& =-R_{43,12}\left(-x_{0}, y_{0} ; B\right) .
\end{aligned}
$$

For the same magnetic field value, the six resistances having a different distribution [i.e., a different $\boldsymbol{\rho}_{0}=\left(x_{0}, y_{0}\right)$ dependence] can be combined to give $R_{\alpha}\left(\boldsymbol{\rho}_{0}, B\right), R_{\beta}\left(\boldsymbol{\rho}_{0}, B\right)$, and $R_{\gamma}\left(\boldsymbol{\rho}_{0}, B\right)$. The resistances, $R_{\alpha}, R_{\beta}, R_{\gamma}$ are symmetric (even function of the magnetic field, see Fig. 4): $R_{\alpha}\left(x_{0} y_{0} ; B\right)=R_{\alpha}\left(x_{0}, y_{0} ;-B\right)$, as a result of the property $\alpha_{21}(B)=\alpha_{12}(-B)$. Moreover, the resistances of the top and the bottom panels of Fig. 4 exhibit the following symmetries, respectively,

$$
R_{\alpha}\left(x_{0}, y_{0} ; B\right)=R_{\alpha}\left(-x_{0},-y_{0} ; B\right)=R_{\alpha}\left(-y_{0},-x_{0} ; B\right),
$$
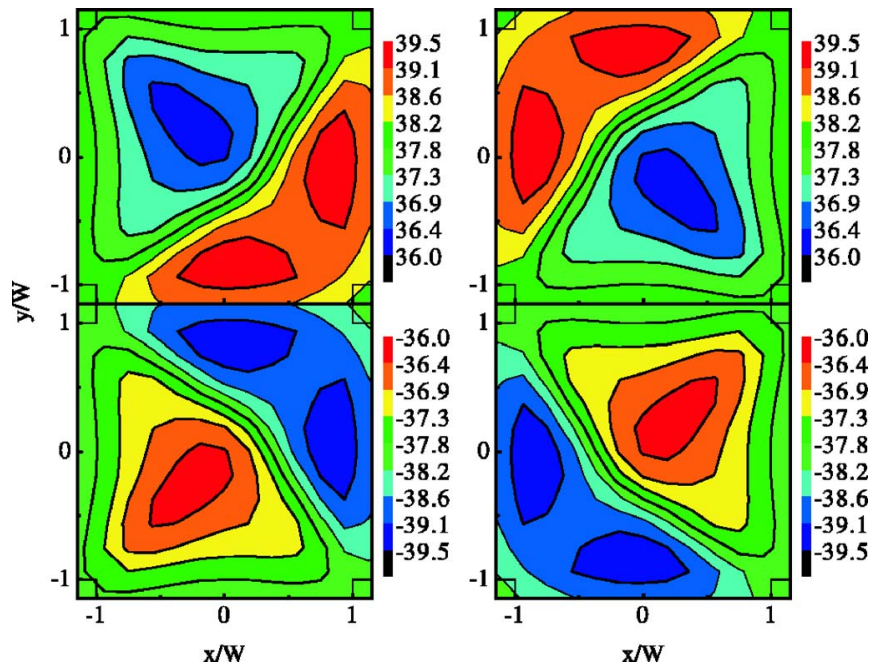

FIG. 3. (Color online) The bend resistances appearing in $R_{\beta}$ (top panels): $R_{14,32}$ (left), $R_{32,14}$ (right) and appearing in $R_{\gamma}$ (bottom panels): $R_{12,43}$ (left), $R_{43,12}$ (right) for $B=50 \mathrm{mT}$ with $V_{0} / E_{F}=0.5$ and $d / W=0.25$.
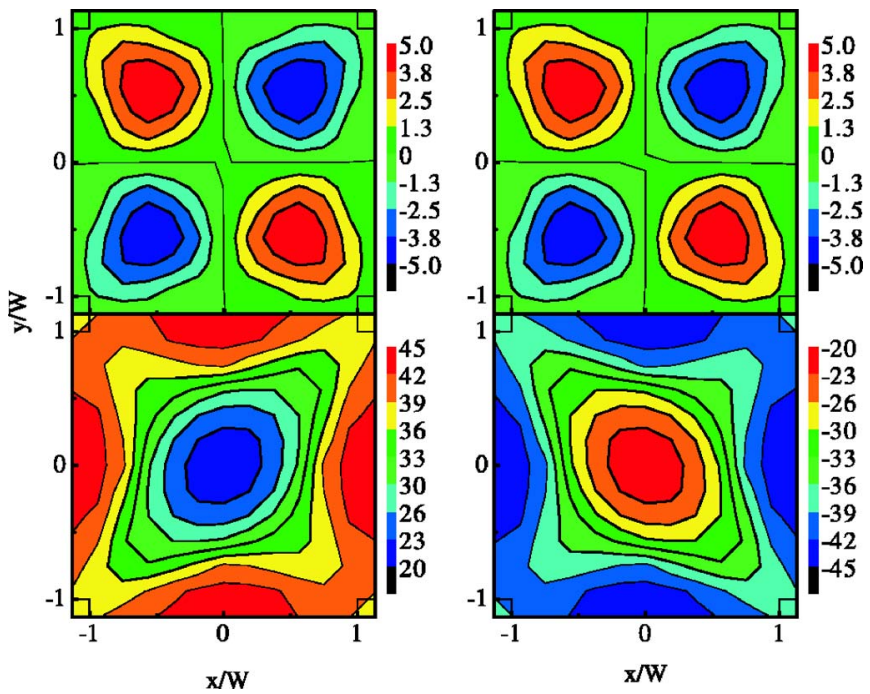

FIG. 4. (Color online) The contour plot of $R_{\alpha}$ (top panels) for $B=50 \mathrm{mT}$ (left), and for $B=-50 \mathrm{mT}$ (right), and the contour plots of $R_{\beta}$ and $R_{\gamma}$ (bottom panels left and right, respectively) for $B=50 \mathrm{mT}$ with $V_{0} / E_{F}=0.98$ and $d / W=0.5$.

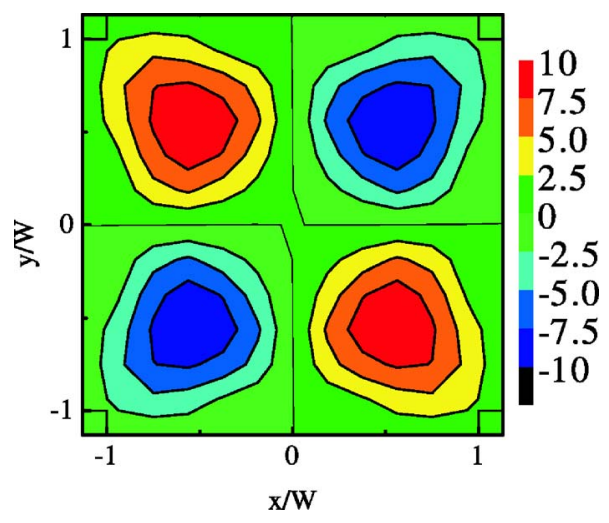

FIG. 5. (Color online) The contour plot of $R_{\alpha}+R_{\beta}+R_{\gamma}$ for $B=50 \mathrm{mT}$ with $V_{0} / E_{F}=0.98$ and $d / W=0.5$. 

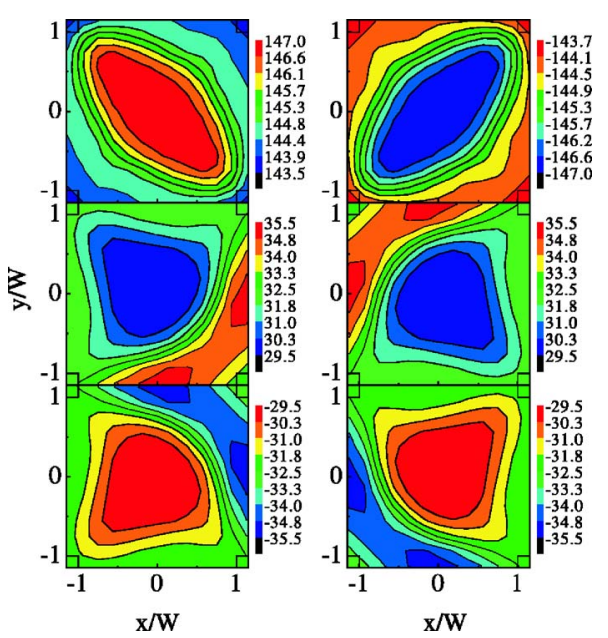

FIG. 6. (Color online) Resistance maps of the terms appearing in $R_{\alpha}$ (top panels): $R_{31,24}$ (left), $R_{24,31}$ (right), the terms in $R_{\beta}$ (middle panels): $R_{14,32}$ (left), $R_{32,14}$ (right) and the terms in $R_{\gamma}$ (bottom panels): $R_{12,43}$ (left), $R_{43,12}$ (right) for $B=100 \mathrm{mT}$ with $V_{0} / E_{F}=0.5$ and $d / W=0.25$.

$$
R_{\beta}\left(x_{0}, y_{0} ; B\right)=-R_{\gamma}\left(-x_{0}, y_{0} ; B\right)=-R_{\gamma}\left(x_{0},-y_{0} ; B\right) .
$$

But importantly, it is clear that the relation $R_{\alpha}+R_{\beta}+R_{\gamma}$ $=0$ is no longer satisfied in every point $\boldsymbol{\rho}_{0}=\left(x_{0}, y_{0}\right)$ as is clearly demonstrated in Fig. 5 where a contour plot of this quantity is plotted. But a less restrictive relation is valid, namely the average, i.e., $\int\left(R_{\alpha}+R_{\beta}+R_{\gamma}\right) d^{2} \rho=0$, is zero.

$(S)$

It is interesting to see what the effect is of an increase of the strength on the magnetic field. In Fig. 6 we show the distribution of resistances for $B=100 \mathrm{mT}$ with $V_{0} / E_{F}=0.5$ and $d / W=0.25$. The top panels contain the terms contained in $R_{\alpha}$, the middle panels those of $R_{\beta}$, and the bottom panels those of $R_{\gamma}$. Notice that the fine structure in the 2D map of $R_{i}\left(x_{0}, y_{0} ; B\right)$ is washed out but the symmetry properties are unaltered. Note also that the absolute values of the Hall (bend) resistances are larger (smaller) than those for the 50 $\mathrm{mT}$ case.

\section{CONCLUSIONS}

In conclusion, we calculated the influence of a local potential barrier on the different resistances of a ballistic mesoscopic Hall bar. The change in resistance depends on the exact position of the potential barrier and symmetry relations were obtained for the $2 \mathrm{D}$ resistance maps. We showed numerically that the Onsager relations and those derived by Büttiker for a four terminal device are also satisfied for the 2D resistances maps. We found that: (1) the bend resistance is more sensitive to a local potential barrier then the Hall resistance and (2) the sensitivity of the Hall bar is larger near some of the corners of the Hall bar. These conclusions are important when such Hall bars are used as local electric field detectors.

\section{ACKNOWLEDGMENTS}

This work was supported by the Belgian Science Policy (IUAP-program) and the Flemish Science Foundation (FWO-Vl).

${ }^{1}$ S. Cunningham, I. A. Larkin, and J. H. Davis, Appl. Phys. Lett. 73, 123 (1998).

${ }^{2}$ T. Trenkler, P. De Wolf, W. Vandervorst, and L. Hellemans, J. Vac. Sci. Technol. B 16, 367 (1998).

${ }^{3}$ M. A. Eriksson, R. G. Beck, M. Topinka, J. A. Katine, R. M. Westervelt, K. L. Campman, and A. C. Gossard, Appl. Phys. Lett. 69, 671 (1996).

${ }^{4}$ A. Baumgartner, T. Ihn, K. Ensslin, G. Papp, F. M. Peeters, K. Maranowski, and A. C. Gossard, Phys. Rev. B 74, 165426 (2006).

${ }^{5}$ B. Baelus and F. M. Peeters, Appl. Phys. Lett. 74, 1600 (1999).

${ }^{6}$ L. Onsager, Phys. Rev. B 38, 2265 (1931).

${ }^{7}$ H. B. G. Casimir, Rev. Mod. Phys. 17, 343 (1945).

${ }^{8}$ M. Büttiker, Phys. Rev. Lett. 57, 1761 (1986).

${ }^{9}$ C. J. W. Beenakker and H. van Houten, in Solid State Physics, edited by H. Ehrenreich and D. Turnbull (Academic, New York, 1991), Vol. 44, p. 98.

${ }^{10}$ C. J. B. Ford, S. Washburn, M. Büttiker, C. M. Knoedler, and J. M. Hong, Phys. Rev. Lett. 62, 2724 (1989).

${ }^{11}$ F. M. Peeters and X. Q. Li, Appl. Phys. Lett. 72, 572 (1998).

${ }^{12}$ A. K. Geim, S. V. Dubonov, I. V. Grigorieva, J. G. S. Lok, J. C. Maan, X. Q. Li, F. M. Peeters, and Yu. V. Nazarov, Superlattices Microstruct. 23, 151 (1998). 